\title{
The Relationship Between COVID-19 Cases and COVID-19 Testing: a Panel Data Analysis on OECD Countries
}

\author{
Umit Cirakli ${ }^{1}$ (D) $\cdot$ Ibrahim Dogan ${ }^{2} \cdot$ Mehmet Gozlu $^{3}$
}

Received: 14 January 2021 / Accepted: 2 April 2021 / Published online: 13 April 2021

(C) The Author(s), under exclusive licence to Springer Science+Business Media, LLC, part of Springer Nature 2021

\begin{abstract}
Testing, one of the methods to combat the COVID-19 outbreak, is highly recommended in all countries. Empirical studies on how testing relates to the control of new cases will help highlight the importance of testing in efforts to combat the epidemic. Therefore, this study aims to investigate the relationship between COVID-19 testing and COVID-19 cases. We use panel autoregressive distributed lag analysis to test the effect of COVID-19 test number on the COVID-19 new cases. The data of the study cover the period from March 19, 2020, to May 01, 2020, for 14 OECD countries. Data were obtained from the https://ourworldindata.org/coronavirus website. According to the results, this study shows that increasing the COVID-19 test number will help to reduce new COVID-19 cases. On the other hand, increase in the test number per thousand will probably not contribute to reducing new COVID-19 cases, because countries do not already test by random selection, and even if they do, it will not contribute to detection and isolating of the new cases without identifying risky groups.
\end{abstract}

Keywords COVID-19 $\cdot$ COVID-19 test number $\cdot$ OECD countries $\cdot$ Panel autoregressive distributed lag model

JEL Classification H0J $\cdot 10 \cdot \mathrm{J} 12 \cdot \mathrm{J} 19$

Umit Cirakli

cirakli@hacettepe.edu.tr

Ibrahim Dogan

ibrahim.dogan@yobu.edu.tr

Mehmet Gozlu

mgozlu@gantep.edu.tr

1 Department of Health Management, Faculty of Economics and Administrative Sciences, Yozgat Bozok University, Yozgat, Turkey

2 Department of Economics, Faculty of Economics and Administrative Sciences, Yozgat Bozok University, Yozgat, Turkey

3 Health Sciences Institute, Gaziantep University, Gaziantep, Turkey 


\section{Introduction}

"We have a simple message for all countries: test, test, test. Test every suspected case. If they test positive isolate them and find out who they have been in close contact with up to 2 days before they developed symptoms and test those people too." (WHO Director-General's opening remarks at the media briefing on COVID-19, 16 March, 2020).

In December 2019, a new infectious disease was detected in Wuhan province of China and it was stated that many people applied to health institutions for this disease in a short time (Wu et al., 2020). In the investigations, it was found that a new coronavirus species mutated from the coronavirus family caused this disease, and in subsequent studies, this virus was named 2019-nCoV', Novel CoV-19, SARSCoV-2, and finally COVID-19 (Kumar et al., 2020: 97; Wu et al., 2020). Due to the high infectious effect of the virus, the disease has been transmitted to many countries in a short time and COVID-19 started to be a global problem especially after the World Health Organization (WHO) declared that COVID-19 outbreak as a global pandemic on March 11, 2020. As a result of rapid contamination, the center of the epidemic became European countries and the USA after a short time.

After the pandemic announcement, the necessity of joint struggle against COVID-19 has been underlined and joint studies have accelerated. Nowadays, where phase 3 studies on vaccination are ongoing, one of the most important methods of preventing COVID-19 cases is to control the spread of the COVID-19 virus by preventing the transmission and destroying local epidemics effectively, strongly, and rapidly (Salathé et al., 2020). In this process, WHO recommends taking certain measures such as quarantine, informing the public about the epidemic, complying with hygiene rules, using personal protective equipment, preparing emergency protocols, comprehensive case finding, rapid testing, emergency isolation of identified cases, meticulous contact monitoring, and strictly close contact with individuals to combat COVID-19 (WHO-China Joint Mission on Coronavirus Disease, 2019: 21-22).

In order to combat COVID-19 effectively, cases must be identified and these people must be quarantined. In order to reduce the quarantines shaped by the incidence of COVID-19, we need to determine who has the infection and who has recovered from the infection (Kumleben et al., 2020). At this point, WHO recommends that diagnostic tests must be performed for detection. In particular, monitoring all confirmed contacts and testing to prevent severe acute respiratory failure is the key to successful prevention of the outbreak. It has been suggested that robust testing, home quarantine, and contact tracing (filming) in combination with strict social isolation can bring COVID-19 cases to controllable levels (Aleta et al., 2020). Testing can also reduce the period of uncertainty associated with cases (Brotherhood et al., 2020: 1). Studies indicate that the incubation period of the disease is five days, and the majority of infected people start to show symptoms within 10-14 days (Fang et al., 2020). Patients who show certain symptomatic symptoms of the epidemic should be tested to confirm the diagnosis of COVID-19 as soon as possible, and those with positive COVID-19 
test results should be isolated from the community and followed up clinically (Peeling et al., 2020: 1). In addition, tests are also very useful in identifying populations at risk and evaluating the effectiveness of control strategies (Peeling et al., 2020). Finally, it is suggested that an effective testing strategy can be used as an important tool in loosening the applied social distance interventions (Aleta et al., 2020). WHO succinctly emphasizes the importance of testing all countries with the following sentence: "We have a simple message for all countries: test, test, test." (WHO Director-General's opening remarks at the media briefing on COVID-19, 16 March 2020).

Some testing approaches can be used to diagnose COVID-19, and these approaches have different advantages and disadvantages (Kumar et al., 2020). The method choiced for testing is usually the real-time reverse transcription-PCR (RT-PCR) test. A major advantage of real-time RT-PCR testing is that the amplification and analysis are performed simultaneously in a closed system to minimize false positive results associated with amplification product contamination (Tang et al., 2020). Although PCR tests give good results, they are expensive and laborious (Kumar et al., 2020). Although PCR tests have high analytical sensitivity, the clinical sensitivity level is not very high because some COVID-19 positive patients cannot be detected due to no having enough viruses in the upper respiratory tract for various reasons (Fang et al., 2020). For this reason, it is very important to determine whether the test correctly detects the cases, even if the diagnostic tests are successfully applied. No diagnostic or screening test is perfect, and false results are inevitable. At this point, it should be taken into consideration that there may be false positive detections or false negative determinations while performing PCR test (Kumleben et al., 2020). It is stated that the highest sensitivity rate of RT-PCR tests is $90 \%$. When all tests are evaluated even at this highest rate, it is obvious that false test results will lead to the spread and increased prevalence of COVID-19 cases. The extent of this negative effect can be better understood, especially when it is considered that these tests are applied in large societies or applied on healthcare personnel (West et al., 2020).

It is recommended the strict application of infection control criteria such as physical distance, hand hygiene, environmental cleaning and disinfection, and especially the use of adequate personal protective equipment, especially by healthcare professionals and patients, to develop risk-stratified protocols for management of false negative test results, determination of risk levels before testing, and the development of accurate diagnostic tests to reduce the negative impact of false negative test results (West et al., 2020: 1128). Therefore, it has been suggested that re-testing may be beneficial in false-negative test results, especially in cases with high clinical suspicion (Fang et al., 2020: 2).

Determining an effective testing policy, taking into account the stated advantages, disadvantages, and limitations, provides various gains in managing the epidemic. Salathé et al. (2020) summarize some of the benefits of testing as followings:

- Helps to keep the COVID-19 virus under control and prevent possible contamination by monitoring and isolating people who come into contact with positively diagnosed people. 
- Testing enables the identification of infectious individuals and groups and allows contact tracing and precautionary self-isolation.

- Provides a more accurate estimate of death rates by confirming the relationship between the onset of the disease and death.

- Testing gives a clear picture of epidemiological situations achieved on the right ground. These data are very important in obtaining mild, severe, and critical patient ratios in order to better manage the problem.

- Testing allows us to get a clear picture of the dynamics of the COVID-19 outbreak.

- Testing allows us to reliably determine the proportion of the population infected with the virus.

On the other hand, even if very good results are obtained by testing, factors such as (i) the people with symptoms are not known exactly, (ii) the test results can take a long time to obtain, (iii) the cost of testing is not negligible (Brotherhood et al., 2020), (iv) insufficient capacity of healthcare infrastructures, (v) failure to detect the disease due to individuals' early days of contracting the virus, and (vi) failure to scale the centers required for testing on a very large scale and not meet the demand (Peeling et al., 2020) can reduce the gains from tests.

Another important point in controlling COVID-19 is the issue of how the test is applied, which greatly affects the effectiveness of the test (Thunström et al., 2020: 2). At this point, different strategies can be followed by the authorities. Some may decide to test only those with symptoms or to test if any disease develops within 14 days from the first contact, and some can test all groups with or without symptoms (Peeling et al., 2020: 1). There are studies involving different views on this subject. For example, Thunström et al. states that the most effective testing policy under a certain time and test limitation is the method of random testing on a voluntary basis (Thunström et al., 2020: 15). Cleevely et al., on the other hand, suggests that the method of periodically testing groups stratified according to risk groups instead of global random testing is the best method of testing under limited resources (Cleevely et al., 2020: 1). Another factor that affects these strategies is the willingness of individuals to have tests. In the study conducted by Thunström et al. (2020: 13), it is stated that factors such as age of individuals, being extrovert and health-related lifestyle characteristics, and their status of having insurance may affect individuals' willingness to have COVID-19 testing (Thunström et al., 2020: 13).

This study is about determining to what extent the testing recommended by WHO affects the case control process. In this regard, this study illustrates that the effect of COVID-19 testing on controlling COVID-19 cases via econometric cointegration test. Our study may provide important contributions to literature. First, since studies about the effects of testing on the number of COVID-19 cases are limited, this study provides evidence to both literature. Since this study will also be an evaluation of the test policies implemented by the countries, it may contribute to the countries making an evaluation in their test policies. Countries are conducting a significant number of tests and trying to increase their testing capacity. Our study provides scientific evidence for their decisions. In addition, in terms of contribution to the literature, it will 
be able to form a basis for cost-effectiveness analyzes that can be made regarding tests, especially in terms of effectiveness measure.

\section{Methods}

\section{Study Design}

To investigate the relationship between COVID-19 test number and COVID-19 case number, panel autoregressive distributed lag (ARDL) cointegration analysis (Panel ARDL Pooled Mean Group estimator) was used which are proposed by Pesaran et al. (1999). This model allows intersection points, short-term coefficients, and error variants to change freely between groups, but keeps the long-term coefficients the same. PMG estimator enables us to investigate long-term homogeneity without implementing the homogeneity of the parameter in the short term (Pesaran et al., 1999). Panel ARDL general equation can be shown as the following regression:

$$
Y_{1, i t}=\alpha_{1 i}+\gamma_{1, i} Y_{1, i t-1}+\sum_{l=2}^{k} \gamma_{1 i} X_{1, i t-1}+\sum_{j=1}^{p-1} \sigma_{1, i j} \Delta Y_{1, i t-j}+\sum_{j=0}^{q-1} \sum_{l=2}^{k} \sigma_{1, i j} \Delta X_{1, i t-j}+\mu_{1, i t}
$$

where " $i$ " is the number of units in the panel, " $\alpha$ " is the constant component of the model, " $\Delta$ " is the first difference operator, and " $k$ " is the number of lags, and " $\mu_{i t}$ " is error term with zero mean and variance constant within each unit.

The stationary of the series lie at the basis of the cointegration analyzes developed for the determination of long-run relationships between time series and variables (Ciftci \& Yildiz, 2015). Therefore, unit root tests for time series were firstly performed. Then, Pedroni Panel Residual Cointegration test (Pedroni, 1999) performed to decide a cointegration relation between the variables. Pedroni (1999) is used to test whether the panel data is cointegrated with 7 different tests and 11 different statistics, under the assumption that there is no cross-sectional dependency. Finally, panel ARDL analysis was conducted in the last stage to estimate the long-run coefficients. Equations of these seven tests proposed by Pedroni (1999) are shown in the following equations:

1. Panel v-Statistic: $T^{2} N^{3 / 2} Z_{\widehat{v} \mathrm{NT}}=T^{2} N^{3 / 2}\left(\sum_{i=1}^{N} \sum_{i=1}^{T} \widehat{L}_{11 i}^{-2} \widehat{e}_{i, t-1}^{2}\right)^{-1}$

2. Panel $t$-Statistic: $Z_{t N T}=\left(\widetilde{\sigma}_{N T}^{2} \sum_{i=1}^{N} \sum_{t=1}^{T} \hat{L}_{11 i}^{-2} \hat{e}_{i, t-1}^{2}\right)^{-\frac{1}{2}} \sum_{i=1}^{N} \sum_{t=1}^{T} \widehat{L}_{11 i}^{-2}\left(\hat{e}_{i, t-1}^{2} \Delta e_{i, t}-\hat{\lambda}_{i}\right)$

3. Panel $p$-Statistic: $T \sqrt{N} Z_{\hat{\rho} N T^{-1}}=T \sqrt{N}\left(\sum_{i=1}^{N} \sum_{i=1}^{T} \widehat{L}_{11 i}^{-2} \hat{e}_{i, t-1}^{2}\right)^{-1} \sum_{i=1}^{N} \sum_{i=1}^{T} \widehat{L}_{11 i}^{-2}\left(\widehat{e}_{i, t-1} \Delta \widehat{e}_{i, t}-\hat{\lambda}_{i}\right)$

4. Panel ADF: $Z_{t N T}^{*}=\left(\widetilde{s}_{N T}^{* 2} \sum_{i=1}^{N} \sum_{t=1}^{T} \widehat{L}_{11 i}^{-2} \widehat{e}_{i, t-1}^{* 2}\right)^{-\frac{1}{2}} \sum_{i=1}^{N} \sum_{t=1}^{T} \widehat{L}_{11 i}^{-2} \widehat{e}_{i, t-1}^{*} \Delta \widehat{e}_{i, t}^{*}$

5. Group t: $N^{-\frac{1}{2}} \bar{Z}_{t N T}=N^{-\frac{1}{2}} \sum_{i=1}^{N}\left(\hat{\sigma}_{i}^{2} \sum_{t=1}^{T} \hat{e}_{i, t-1}^{2}\right)^{-\frac{1}{2}} \sum_{t=1}^{T}\left(\hat{e}_{i, t-1} \Delta \widehat{e}_{i, t}-\hat{\lambda}_{i}\right)$

6. Group p: $N^{-\frac{1}{2}} \bar{Z}_{t N T}=T N^{-\frac{1}{2}} \sum_{i=1}^{N}\left(\sum_{t=1}^{T} \hat{e}_{i, t-1}^{2}\right)^{-1} \sum_{t=1}^{T}\left(\hat{e}_{i, t-1} \Delta \hat{e}_{i, t}-\hat{\lambda}_{i}\right)$

7. Group ADF: $N^{-\frac{1}{2}} \bar{Z}_{t N T}^{*}=N^{-\frac{1}{2}} \sum_{i=1}^{N}\left(\sum_{t=1}^{T}{\tilde{s}_{i}^{* 2}}^{* 2} \widehat{e}_{i, t-1}\right)^{-\frac{1}{2}} \sum_{t=1}^{T} \hat{e}_{i, t-1} \Delta \widehat{e}_{i, t}$ 
After detecting cointegration relationship between series, we conducted panel ARDL analysis to estimate long-run and short-run relations between series. We used five different ARDL models to ensure robustness of the relationships. These five models are shown in the following equations:

\section{Model 1.}

$$
\begin{aligned}
\Delta L N C_{i t}= & \beta_{0}+\sum_{j=1}^{p i} \lambda_{i j} \Delta L N C_{i, t-j}+\sum_{j=0}^{q i} \delta_{i j} \Delta L T T_{i, t-j}+\sum_{j=0}^{q i} \varphi_{i j} \Delta L N T_{i, t-j} \\
& +\sum_{j=0}^{q i} \theta_{i j} \Delta L T T \text { Ter } T_{i, t-j}+\sum_{j=0}^{q i} \ddot{\theta}_{i j} \Delta L N T \operatorname{Ler} T_{i, t-j}+\gamma_{1} L N C_{i, t-1}+\gamma_{2} L T T_{i, t-1} \\
& +\gamma_{3} L N T_{i, t-1}+\gamma_{4} L T T \text { TerT } \\
i, t-1 & +\gamma_{5} L N T p e r T_{i, t-1}+\varepsilon_{i t}
\end{aligned}
$$

\section{Model 2.}

$$
\begin{aligned}
\Delta L N C_{i t}= & \beta_{0}+\sum_{j=1}^{p i} \lambda_{i j} \Delta L N C_{i, t-j}+\sum_{j=0}^{q i} \delta_{i j} \Delta L T T_{i, t-j}+\sum_{j=0}^{q i} \varphi_{i j} \Delta N T_{i, t-j}+\gamma_{1} L N C_{i, t-1} \\
& +\gamma_{2} L T T_{i, t-1}+\gamma_{3} L N T_{i, t-1}+\varepsilon_{i t}
\end{aligned}
$$

\section{Model 3.}

$$
\begin{aligned}
\Delta L N C_{i t}= & \beta_{0}+\sum_{j=1}^{p i} \lambda_{i j} \Delta L N C_{i, t-j}+\sum_{j=0}^{q i} \delta_{i j} \Delta L T T_{i, t-j}+\sum_{j=0}^{q i} \varphi_{i j} \Delta \operatorname{LTTperT}_{i, t-j}+\gamma_{1} L N C_{i, t-1} \\
& +\gamma_{2} L T T_{i, t-1}+\gamma_{3} \operatorname{LTTperT}_{i, t-1}+\varepsilon_{i t}
\end{aligned}
$$

\section{Model 4.}

$$
\begin{aligned}
\Delta L N C_{i t}= & \beta_{0}+\sum_{j=1}^{p i} \lambda_{i j} \Delta L N C_{i, t-j}+\sum_{j=0}^{q i} \delta_{i j} \Delta L N T_{i, t-j}+\sum_{j=0}^{q i} \varphi_{i j} \Delta L N T p e r T_{i, t-j}+\gamma_{1} L N C_{i, t-1} \\
& +\gamma_{2} L N T_{i, t-1}+\gamma_{3} L N T p e r T_{i, t-1}+\varepsilon_{i t}
\end{aligned}
$$




\section{Model 5.}

$$
\begin{aligned}
\Delta L N C_{i t}= & \beta_{0}+\sum_{j=1}^{p i} \lambda_{i j} \Delta L N C_{i, t-j}+\sum_{j=0}^{q i} \delta_{i j} \Delta L T T_{i, t-j}+\sum_{j=0}^{q i} \varphi_{i j} \Delta \operatorname{LNTperT}_{i, t-j}+\gamma_{1} L N C_{i, t-1} \\
& +\gamma_{2} L T T_{i, t-1}+\gamma_{3} L N T p e r T_{i, t-1}+\varepsilon_{i t}
\end{aligned}
$$

In the above equations, " $\beta_{0}$ " is the constant component of the model, " $\Delta$ " is the first difference operator, " $\mathrm{L}$ " is the logarithm of the series, "NC" is new COVID-19 cases, "TT" is total tests, "NT" is new tests, "TTperT" is total tests per thousand, "NTperT" is new tests per thousand, and " $\varepsilon_{i t}$ " is error term with zero mean and variance constant within each unit. We used the logarithm of series in the analysis, because it is possible to interpret the results as elasticity when the logarithms of the series are used.

\section{Data and Analysis}

The data of the study were obtained as daily data for 14 OECD countries spanning 44 days from March 19, 2020, through May 01, 2020, from the https://ourworldindata. org/coronavirus website. Variables of study are shown in Table 1. Fourteen OECD countries include Turkey, USA, Belgium, Finland, England, Switzerland, Italy, South Korea, Hungary, Mexico, Poland, Portugal, Slovenia, and New Zealand. Selection of these countries and data dates is made according to the following criteria:

- Being an OECD country,

- Having complete data for specified dates

All analyzes were carried out in Eviews 9.5 package program which is frequently used in econometric analysis.

\section{Results}

\section{Results of Unit Root Tests}

In this study, we used a variety of tests to determine the stationarity of the time series in panel datasets: Levin, Lin, and Chu test (Levin et al., 2002); Im, Pesaran,

Table 1 Variables and abbreviations

\begin{tabular}{ll}
\hline Variables & Abbreviation \\
\hline New cases & NC \\
News tests & NT \\
New tests per thousand & NTperT \\
Total tests per thousand & TTperT \\
Total tests & TT \\
\hline
\end{tabular}


and Shin test (Im et al., 2003); ADF-Fisher chi-square test (Maddala \& Wu, 1999); and PP-Fisher chi-square test (Choi, 2001). The results of unit root tests were shown in the Table 2. According to the unit test results, it is seen that all the variables meet the condition of being stationary in their level values or their first difference values.

\section{The Results of Panel Cointegration Tests}

In this section, we used the Pedroni Panel Residual Cointegration test (Pedroni, 1999) to decide a cointegration relation between the variables. The Pedroni Panel Residual Cointegration test includes 7 different tests and 11 different statistics to determine the cointegration relationship between variables, under the assumption that there is no cross-sectional dependency (Pedroni, 1999).

The results of Pedroni Panel Residual Cointegration test were shown in Table 3. From the results shown in table, 10 out of 11 different statistics show a significant long-run cointegration relationship between series. Therefore, we can conduct panel ARDL analysis to estimate long-run and short-run coefficients.

\section{The Results of Panel ARDL Analysis}

The results of panel ARDL analysis were shown in Table 4. The selection of the most appropriate ARDL model was made automatically by the Eviews 9.5 package program according to the Akaike information criterion and the maximum lag length of 4 with automatic selection.

As seen in Table 4, we used "logarithmic number of new cases" as dependent variable and, "the logarithmic number of total tests, number of new tests, number of total tests per thousand, and number of new tests per thousand" as estimators. From the results of panel ARDL analysis in Table 4, it was found that there was a significant negative relationship between the number of total tests and new COVID-19 cases in ARDL Model 1, which includes all the estimators. The other estimators in ARDL Model 1 were not significantly correlated with dependent variable. In ARDL Model 2, it was found that the relationship between the number of new tests and the number of new COVID-19 cases was significantly positive, while there was significant negative relationship between the number of total tests and the number of new COVID-19 cases. The results of ARDL Model 3 show that while the relationship between the number of total tests and the number of new COVID-19 cases is significantly negative, the number of total test per thousand and the number of new COVID-19 cases are significantly positively correlated. ARDL Model 4 indicates that there is not any significant relationship between the number of new tests, the number of new tests per thousand and the number of new COVID-19 cases. Finally, while the relationship between the number of total tests and the number of new COVID-19 cases is significantly negative, the relationship between the number of new tests per thousand and the number of new COVID-19 cases is significantly positive in ARDL Model 5. 


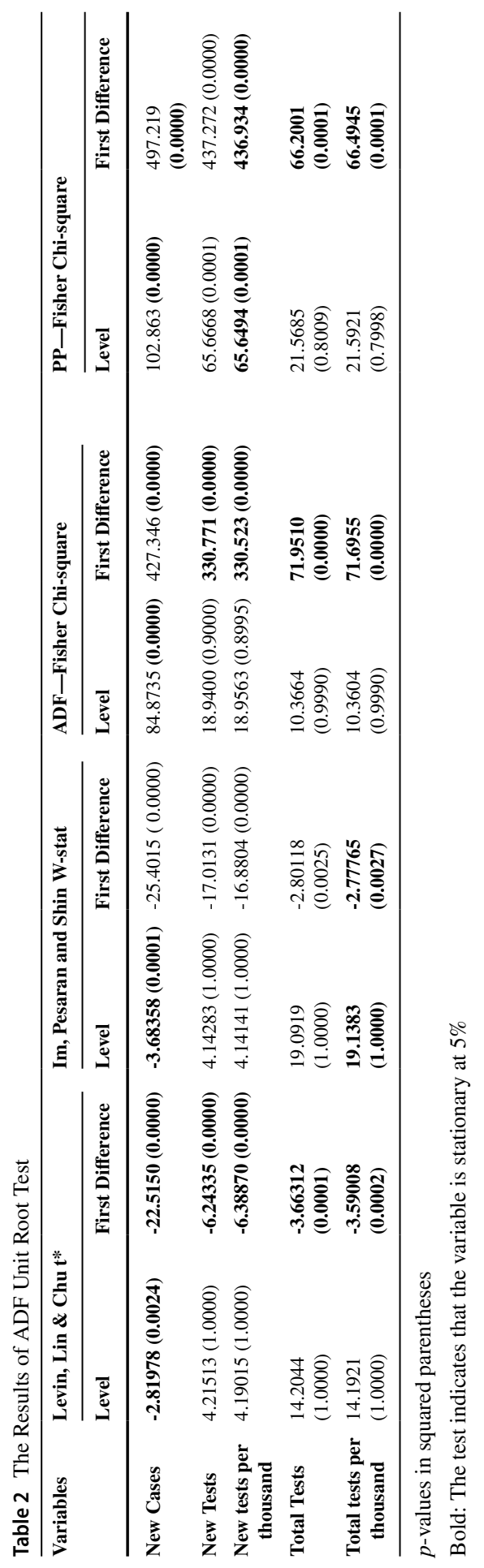


Table 3 The results of Pedroni Panel Residual Cointegration tests

Included observations: 616

Cross-sections included: 14

Null Hypothesis: No cointegration

Trend assumption: No deterministic trend

User-specified lag length: 1

Newey-West automatic bandwidth selection and Bartlett kernel

\begin{tabular}{lllll}
\hline & & & Weighted & \\
& Statistic & Prob & Statistic & Prob \\
Panel v-Statistic & $1.747 * *$ & 0.040 & -1.431 & 0.9239 \\
Panel rho-Statistic & $-7.743 * * *$ & 0.000 & $-5.567 * * *$ & 0.0000 \\
Panel PP-Statistic & $-12.277 * * *$ & 0.000 & $-10.527 * * *$ & 0.0000 \\
Panel ADF-Statistic & $-3.161 * * *$ & 0.000 & $-4.622 * * *$ & 0.0000 \\
Alternative hypothesis: individual AR coefs. (between-dimension) & & \\
& Statistic & Prob & & \\
Group rho-Statistic & $-5.577 * * *$ & 0.000 & & \\
Group PP-Statistic & $-12.717 * * *$ & 0.000 & & \\
Group ADF-Statistic & $-3.881 * * *$ & 0.000 & & \\
\hline
\end{tabular}

*Significant cointegration relationship at 5\%; ***Significant cointegration relationship at $1 \%$

Since we can interpret the coefficients as elasticity, coefficients of total tests show that a $1 \%$ increase in the number of total tests will cause a $1.3 \%$ decrease in the number of new cases for model 1 , a $1.7 \%$ decrease in the number of new cases for model 2 , a $1.04 \%$ decrease in the number of new cases for model 3 , and a $1.7 \%$ decrease in the number of new cases for model 4 . Finally, it is possible to say that a $1 \%$ increase in the number of total tests will result a $1.45 \%$ decrease on average in the number of new cases for four significant models.

In addition to panel ARDL analysis, we used the Hausman test to ensure that the PMG estimator is adequate for ARDL Model 1, which is the main model. The results of Hausman test in Table 5 suggest that PMG estimator is adequate for the other models. 


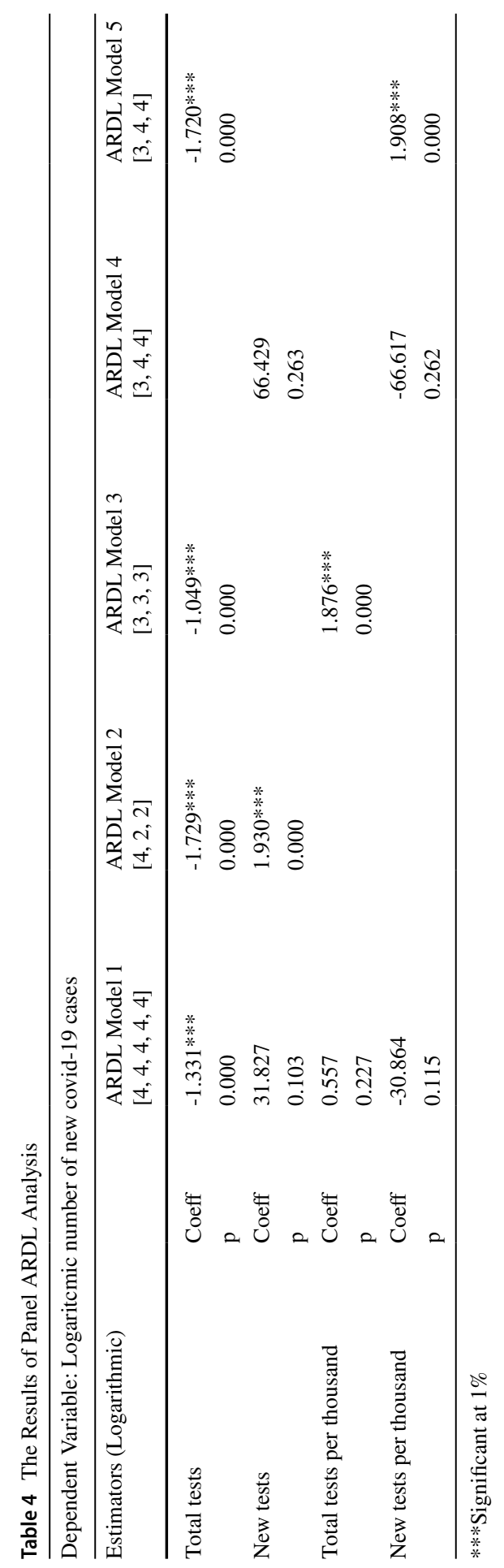


Table 5 The results of Hausman test

\begin{tabular}{llll}
\hline \multicolumn{3}{l}{ Correlated random effects-Hausman test } & \\
\cline { 1 - 2 } Models & Chi-sq. statistic & Chi-sq. d.f & Prob \\
\hline Model 1 & 6.850279 & 4 & 0.1440 \\
Model 2 & 4.886360 & 2 & 0.0869 \\
Model 3 & 1.760451 & 2 & 0.4147 \\
Model 4 & 3.130548 & 2 & 0.2090 \\
Model 5 & 5.966332 & 2 & 0.0506 \\
\hline
\end{tabular}

\section{Conclusions and Discussions}

The aim of this study is to reveal the relationship between COVID-19 test number and COVID-19 case number. In this context, we analyzed 44-day COVID-19 data including new cases, total tests, new tests, total test per thousand, and new tests per thousand from March 19, 2020, through May 01, 2020, for 14 OECD countries by panel ARDL model. According to the results, there were significantly negative relationship between number of total tests and new COVID-19 cases in all ARDL models including number of total tests. From the coefficients of total tests, it is possible to say that $1 \%$ increase in the total number of tests will reduce the number of new cases by $1.45 \%$ on average for four significant models. On the other hand, results show that the number of new tests and new tests per thousand are correlated positively with new COVID-19 cases only in one model of 3 models; the relationship in the other models are negative but not significant. Such a result is expected as the probability of determining new cases increases with the increase in the number of new tests. In addition, the countries do not usually test for COVID-19 by random selection. Lastly, the relationship between new COVID-19 cases and the number of total tests per thousand is positive in two models but only one is significant.

In conclusion, this study shows that increasing the COVID-19 test number can help to reduce new COVID-19 cases. On the other hand, increase in the test number per thousand can probably not contribute to reducing new COVID-19 cases, because countries do not already test by random selection, and even if they do, it cannot contribute to detection and isolating of the new cases without identifying risky groups. Although this study shows increasing the number of tests can contribute to reducing new COVID-19 cases, it has some limitations. First of all, we only used data for 14 OECD countries with full data. Therefore, the results cannot be generalized for all OECD countries. There are also many other factors that have contributed to the decrease in the number of new cases. In this contest, since this study uses only the number of tests, the results are valid with the assumption that other factors are constant. For this reason, more robust results can be obtained in a study that includes other factors, if appropriate data can be obtained.

Country authorities are increasing and trying to increase the number of COVID19 tests day by day. There is a cost of the test against these increasing efforts. When calculating according to reimbursement rates to hospitals for tests in Turkey, during the period of study for tests of 14 OECD countries, the average daily cost of 
270,740 US Dollars arises for each country. Against this cost, knowing whether testing reduces the number of cases will provide the basis for more realistic policy decisions. Our study with the result of the reducing effect of COVID-19 testing on the number of new cases provides statistical evidence for political decisions. Increasing test number will also contribute to country's economy indirectly. In particular, increasing the number of tests within a well-designed plan can help the country survive economically in the pandemic environment by increasing the contribution of the workforce to the economy.

Our study shows the significant decreasing effect of the number of tests. In order to increase the effectiveness of the test, testing people at risk may be more effective and may contribute to reducing the cost. In order to increase the effectiveness of the test, it is also recommended to update the test algorithms regarding who, in which situations, and how to perform the tests. Increasing the number of tests can help reduce the number of cases and improve the economy by increasing the workforce contribution. However, it is recommended to make this increase with well-designed test algorithms rather than an unplanned increase.

For future studies, this study has taken into account only the test numbers and the number of cases. Econometric studies may be conducted in which conditions such as hygiene conditions, travel restrictions, providing physical distance and curfew are evaluated together. In addition, this study may provide a basis for studies with appropriate data that can reveal the cost-effectiveness of the tests. Countries can also examine the impact of tests on reducing the number of cases by identifying risky people with the filiation teams.

Funding We declare that no funding wasreceived for this article.

\section{Declarations}

Research Involving Human and Animal Participants This article does not contain any studies with human participants or animals performed by any of the authors.

Conflict of Interest Authors have no potential conflict of interest.

\section{References}

Aleta, A., Martín-Corra, D., Piontti, A. P., Ajelli, M., Litvinovar, M., et al. (2020). Modelling the impact of testing, contact tracing and household quarantine on second waves of COVID-19. Nature Human Behaviour, 4, 964-971

Brotherhood, L., Kircher, P., Santos, C. \& Tertilt, M. (2020). An economic model of the Covid-19 epidemic: The importance of testing and age-specific policies. CESifo Working Papers, Munich: Munich Society for the Promotion of Economic Research - CESifo GmbH

Ciftci, F., \& Yildiz, R. (2015). The economic determinants of foreign direct investment: A time series analysis on the Turkish economy. Business and Economics Research Journal, 6(4), 71-95

Cleevely, M., Susskind, D., Vines, D., Vines, L., \& Wills, S. (2020). A workable strategy for COVID-19 testing: Stratified periodic testing rather than universal random testing. Oxford Review of Economic Policy, 36(Supplement_1), 1-24 
Choi, I. (2001). Unit root tests for panel data. Journal of International Money and Finance, 20(2), 249-272

Fang, F.C., Naccache S.N., Greninger A.L. (2020) The laboratory diagnosis of coronavirus disease 2019-Frequently asked questions. Clinical Infectious Diseases.

Im, K. S., Pesaran, M. H., \& Shin, Y. (2003). Testing for unit roots in heterogeneous panels. Jounal of Econometrics, 115, 53-74

Kumar, R., Nagpal, S., Kaushik, S., \& Mendiratta, S. (2020). COVID-19 diagnostic approaches: Different roads to the same destination. Virus Disease, 31(2), 97-105

Kumleben, N., Bhopal, R., Czypionka, T., Gruer, L., Kock, R., Stebbing, J., \& Stigler, F. L. (2020). Test, test, test for COVID-19 antibodies: The importance of sensitivity, specificity and predictive powers. Public Health, 185, 88-90. https://doi.org/10.1016/j.puhe.2020.06.006

Levin, A., Lin, C.-F., \& Chu, C.-S.J. (2002). Unit root tests in panel data: Asymptotic and finite sample properties. Jounal of Econometrics, 108, 1-24

Maddala, G. S., \& Wu, S. (1999). A comparative study of unit root tests with panel data and a new simple test. Oxford Bulletin of Economics and Statistics, 61(S1), 631-652

Peeling, R. W., Wedderburn, C. J., Garcia, P. J., Boeras, D., Fongwen, N., Nkengasong, J., \& Heymann, D. L. (2020). Serology testing in the COVID-19 pandemic response. The Lancet Infectious Diseases

Pedroni, P. (1999). Critical values for cointegration tests in heterogeneous panels with multiple regressors. Oxford Bulletin of Economics and Statistics, 61(S1), 653-670

Pesaran, M. H., Shin, Y., \& Smith, R. P. (1999). Pooled mean group estimation of dynamic heterogeneous panels. Journal of the American Statistical Association, 94(446), 621-634

Salathé, M., Althaus, C. L., Neher, R., Stringhini, S., Hodcroft, E., Fellay, J., et al. (2020). COVID-19 epidemic in Switzerland: On the importance of testing, contact tracing and isolation. Swiss medical weekly, 150(11-12), w20225

Tang, Y. W., Schmitz, J. E., Persing, D. H., \& Stratton, C. W. (2020). Laboratory diagnosis of COVID-19: Current issues and challenges. Journal of clinical microbiology, 58(6)

Thunström, L., Ashworth, M., Shogren, J. F., Newbold, S., \& Finnoff, D. (2020). Testing for COVID-19: Willful ignorance or selfless behavior?. Behavioural Public Policy, 1-26

Wu, F., Zhao, S., Yu, B., Chen, Y. M., Wang, W., Song, Z. G., \& Yuan, M. L. (2020). A new coronavirus associated with human respiratory disease in China. Nature, 579(7798), 265-269

West, C. P., Montori, V. M., \& Sampathkumar, P. (2020). COVID-19 testing: The threat of false-negative results. In Mayo Clinic Proceedings (Vol. 95, No. 6, pp. 1127-1129). Elsevier

World Health Organization-China Joint Mission on Coronavirus Disease. (2019). Report of the WHO China Joint Mission on Coronavirus Disease 2019 (COVID-19). https://www.who.int/docs/defaultsource/coronaviruse/who-china-joint-mission-on-covid-19-final-report.pdf. [Accessed 1 April 2020]

Publisher's Note Springer Nature remains neutral with regard to jurisdictional claims in published maps and institutional affiliations. 